\title{
The Lateral Thoracodorsal Flap in Delayed Bilateral Breast Reconstruction for an Obese Patient with Redundant Lateral Chest Wall Soft Tissue
}

\author{
Jin Su Shin, Eun Soo Park, \\ Yong Bae Kim, Seung Min Nam \\ Department of Plastic and Reconstructive \\ Surgery, College of Medicine, \\ Soonchunhyang University, Bucheon, \\ Korea
}

This work was supported by the Soonchunhyang University Research Fund.

No potential conflict of interest relevant to this article was reported.

\begin{abstract}
Obesity (body mass index $[\mathrm{BMI}] \geq 30 \mathrm{~kg} / \mathrm{m}^{2}$ ), one of the main risk factors for complications of breast reconstruction, makes surgeon hesitate to choose a single ideal surgical technique. Compared to transverse rectus abdominis myocutaneous flap and latissimus dorsi flap, standard methods for autologous breast reconstruction, local flaps have some advantages in terms of aesthetic results and surgical morbidity. A 55-year-old woman who had been undergone modified radical mastectomy, complaints of breast deformity and redundant both lateral chest wall soft tissue. We decided to perform the lateral thoracodorsal flap with implant considering obesity which can cause prolonged operating time and unpredictable results. No major complications were encountered and competent result was obtained when cosmetic analysis was conducted 6 months after surgery.
\end{abstract}

Keywords Breast reconstruction, Flap, Obesity, Cosmetic

\section{INTRODUCTION}

Over the past 30 years, surgical methods for breast reconstruction have evolved and numerous studies have been conducted with the aim of improving aesthetic goals. However, risk factors such as smoking, diabetes, huge breast increase the postoperative complication that causes unsatisfactory result to both surgeon and patient $[1,2]$. Obesity (body mass index $[\mathrm{BMI}] \geq 30 \mathrm{~kg} / \mathrm{m}^{2}$ ), one of the main risk factors, not only leads to postoperative reconstruction failure but also prolongs the operating time. It makes surgeon hesitate to choose a single ideal surgical technique.

Compared to transverse rectus abdominis myocutaneous flap and latissimus dorsi flap, standard methods for autologous breast reconstruction, local flaps have some advantages in terms of aes-

Received: Jan 26, 2015 Revised: Feb 17, 2015 Accepted: Feb 18, 2015 Correspondence: Eun Soo Park Department of Plastic and Reconstructive Surgery, Soonchunhyang University Bucheon Hospital, 170 Jomaru-ro, Wonmi-gu, Bucheon 420-767, Korea. E-mail: peunsoo@schmc.ac.kr

Copyright @ 2015 The Korean Society for Aesthetic Plastic Surgery.

This is an Open Access article distributed under the terms of the Creative Commons Attribution Non-Commercial License (http://creativecommons.org/licenses/by-nc/3.0/) which permits unrestricted non-commercial use, distribution, and reproduction in any medium, provided the original work is properly cited. www.e-aaps.org thetic results and surgical morbidity. The technique is simpler that reduces the operating time, the surgery is less aggressive, the skin color and texture are similar, and the patient has a shorter recovery period. Described as a fasciocutaneous flap in the 1980s, the lateral thoracodorsal flap is a well studied procedure for late breast reconstruction following radical surgery. It relies on the redundancy of skin that can be pinched together without transferring tension on the mammary region [1-4]. We report the case of bilateral thoracodorsal flap with cohesive gel implant performed to woman who had redundant lateral chest wall soft tissue after modified radical mastectomy. We demonstrated the successful use of the technique, which achieved satisfactory result improving the contour of lateral chest wall.

\section{CASE}

\section{Patient}

A 55-year-old woman who had been diagnosed bilateral breast cancer and received modified radical mastectomy 4 years ago, complaint of breast deformity and redundant both lateral chest wall soft tissue after surgery (Fig. 1). Linear shaped, $13 \mathrm{~cm}$ sized scars left on breast and she was in a state of obesity: BMI $31.2 \mathrm{~kg} / \mathrm{m}^{2}(155$ $\mathrm{cm} / 75 \mathrm{~kg}$ ). Since the patient had obesity and lateral hooding, our 


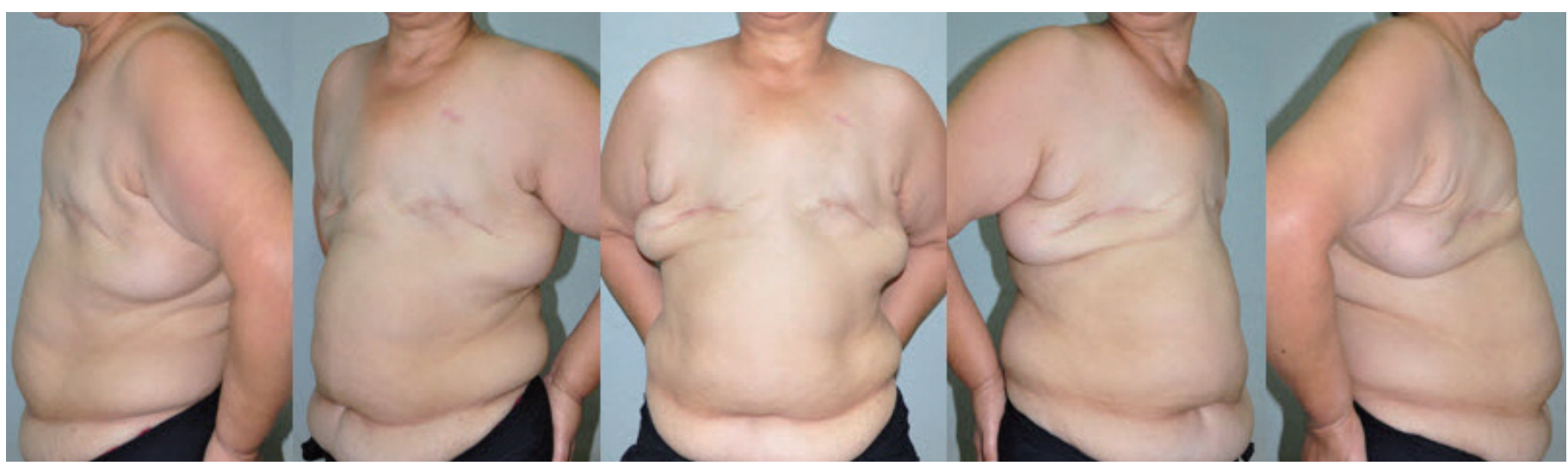

Fig. 1. Contour deformity of both breast with scars and redundant lateral chest wall soft tissue.

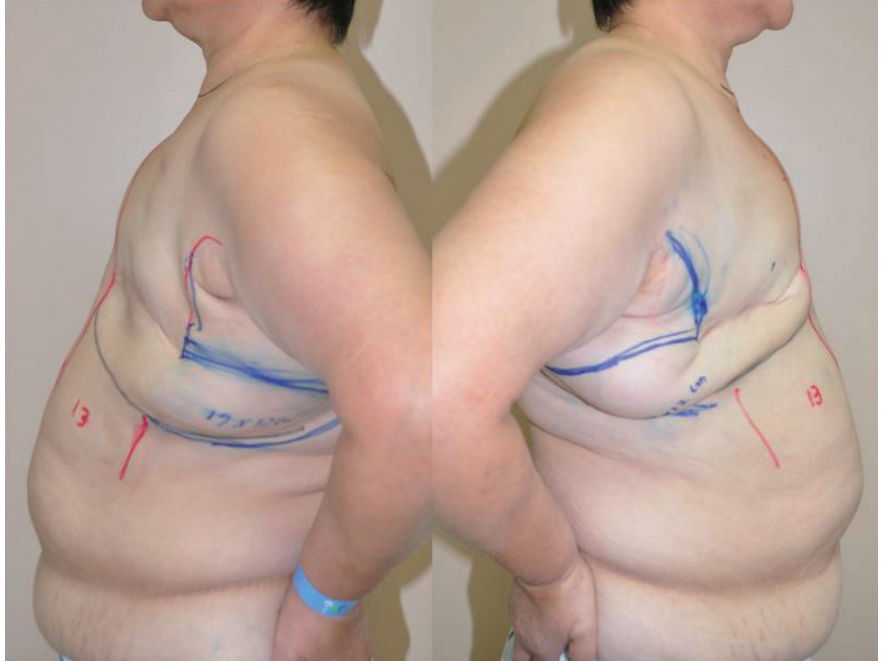

Fig. 2. Preoperative view of the designed lateral thoracodorsal flap.

concern was about taking long time of surgery and postoperative complications.

We decided to use an alternative method using the lateral thoracodorsal flap which is simple and reliable in order to reduce complication and correct the lateral hooding.

\section{Surgical technique}

In both lateral thoracic area, a wedge-shape flap was designed, applying the pinch test, which uses the thumb and index finger, to measure the remaining tissue. The axis of the flap was set along the line of the inframammary fold, extending toward the lateral and posterior sides, and the base of the flap was designed to lie on the anterior axillary line so that the postoperative scar could be hidden under the brassiere line. The base of the flap was set at $12 \mathrm{~cm}$, as determined by the pinch test $(3 \mathrm{~cm})$, and the length of the flap was measured to be $17 \mathrm{~cm}$ (Fig. 2).

The lateral thoracodorsal flap is a medially-based fasciocutaneous flap raised from the upper lateral thoracic region with its long

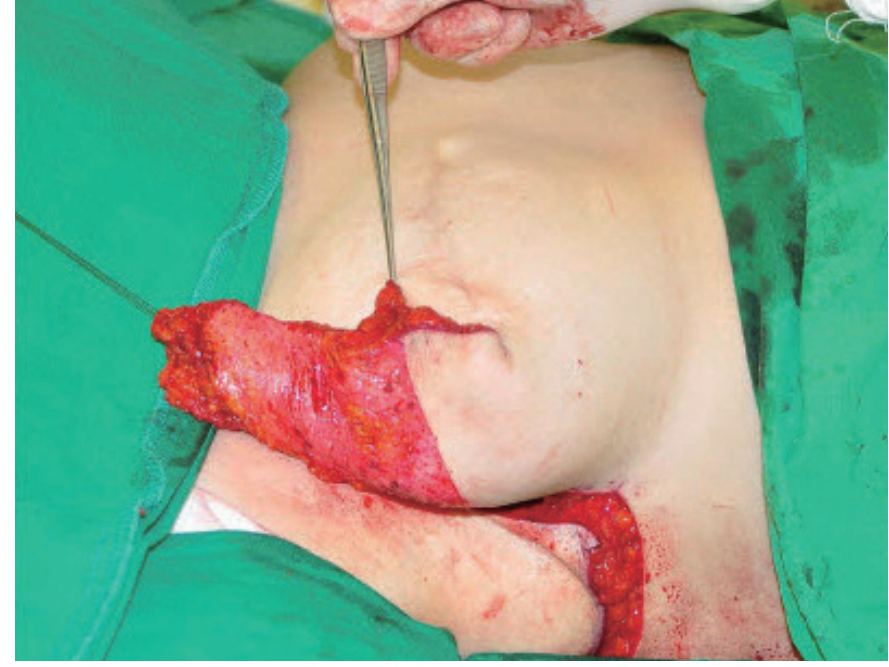

Fig. 3. The flap with de-epithelization of excessive skin is raised from the lateral and dorsal aspects of the thoracic wall.

axis extending laterally and dorsally from the level of the submammary crease along the sixth and seventh ribs. The distal third of the flap over-rides the anterior border of the latissimus dorsi muscle and the base is situated above the serratus muscle at the level of the anterior axillary line.

In the lateral thoracic area, a wedge-shape flap was designed, applying the pinch test, which uses the thumb and index finger, to measure lateral chest wall soft tissue. The flap margin incision was carried down to the underlying anterior serratus and latissimus dorsi muscles. The skin and the subcutaneous fat were dissected from the underlying muscles in a lateral to medial direction. Caution was taken to include the fascia from the superficial to the deep layer during dissection avoiding a wide undermining in the inframammary sulcus. The flap was transposed into the lateral part of the mastectomy region in an almost perpendicular cranial manoeuvre, separating a horizontal or an oblique mastectomy scar line and stapled temporarily on the skin; both breasts were compared 


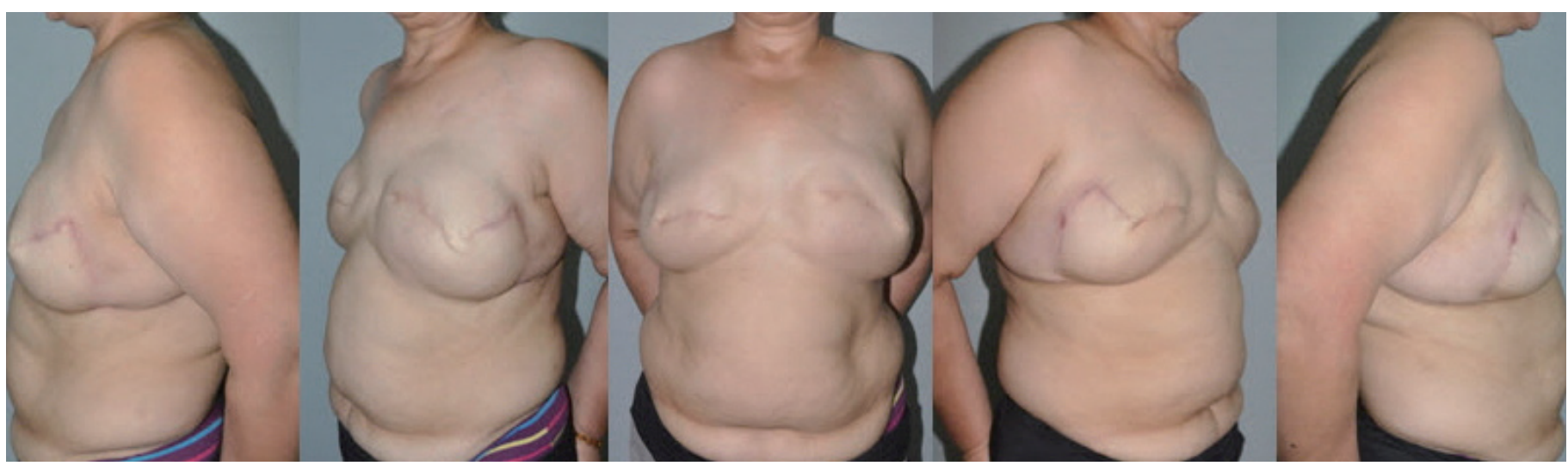

Fig. 4. Postoperative reconstruction state; shape of the breast with lateral hooding was improved.

in the lying and sitting positions to determine the mold of the flap and shape of the breast, and the excessive skin $(12 \times 9 \mathrm{~cm})$ was deepithelized (Fig. 3) after being transferred, the tip of the flap covers pectoralis major in the anterior axillary fold. A wide subpectoral pocket was created by detaching the costal and lower sternal origin of pectoralis major. Anatomically textured implant (Allergan 410FM) was inserted under the detached part of pectoralis major, but the lower lateral part of the implant was covered only by the lateral thoracodorsal flap. Each volume of implant was $420 \mathrm{cc}$ (height $12.5 \mathrm{~cm}$, width $13.5 \mathrm{~cm}$, projection $5.3 \mathrm{~cm}$ ). It took 4 hours 40 minutes to finish the surgery on both breast.

\section{RESULT}

Small skin necrosis $(1 \times 1.5 \mathrm{~cm})$ developed in the flap tip of the left breast in the early post operative period, but it was healed with conservative treatment. The contour deformity of breast with lateral hooding was improved in immediate finding. The cosmetic analysis was conducted with the patients at 6 months postoperatively and it showed that the patient was satisfied with the result. The bulging on lateral chest wall was almost disappeared (Fig. 4).

\section{DISCUSSION}

Basically, local lateral flaps used a rotation or transposition flap of skin and subaxillary fat into the breast defect. In 1996, Clough et al. [5] described a rotation flap of redundant subaxillary skin into medium sized breast defects. Similarly, Kroll and Singletary [6] transferred the subaxillary skin and subcutaneous fat as a rotation flap to reconstruct a lateral breast defect. According to the defect location, the pedicle flap may be based superiorly or inferiorly and shifted to the defect into the less conspicuous area below the axilla.

Lateral thoracodorsal flap is a wedge-shaped fasciocutaneous transposition flap raised from the infra-axillary lateral flank region, which was first introduced by Holmström and Lossingas [1] an im- plant-based technique using local flaps for delayed postmastectomy breast reconstruction in 1986. Woerdeman et al. [2] later used lateral thoracodorsal flaps for immediate breast reconstruction; expanded its application to subjects by de-epithelization of the flap; investigated risk factors such as patient age, obesity, medical history, and smoking; and reported its usefulness and precautions. Since then other groups have continually reported on their clinical experience with lateral thoracodorsal fasciocutaneous flap breast reconstruction $[2,7,8]$. All of them observed that the procedure, associate with an implant, is useful for the delayed breast reconstruction.

The flap complication took place in the initial postoperative period for this patient but no major complications were encountered. Woerdeman et al. [2] observed that flap-related complications occurred in 18.3 percent of cases, with an 11.6 percent rate of partial necrosis and a 3.3 percent rate of fat necrosis. Moreover, the suggested length of the flap should be kept shorter than $17 \mathrm{~cm}$ considering the statistical significance between partial flap necrosis and flap length [9].

The lateral thoracodorsal flap procedure has the advantage of being similar in color and texture to the native breast; in addition, morbidity of the donor site is minimized without sacrificing muscles or nerves, implying less invasiveness, a shorter hospital stay, and good aesthetic and functional results [1-4,7,8]. Furthermore, it could reduce the operating time in case of both breast surgery or patients with obesity. Compared with other reconstructive techniques that involve rearrangement of glandular tissue, the lateral thoracodorsal fasciocutaneous flap does not alter the normal architecture of the breast and the original tumor site location [4].

Despite its main benefits, the lateral thoracodorsal flap has some disadvantage of poor vascularization to the most distant parts because the flap is not an axial flap. This situation can predispose to partial necrosis and an undesirable result. Care must be taken in high-risk patients such as smokers and patients with associated comorbid diseased. In addition, it is prudent to evaluate dermal bleeding in the tip of the flap and shorten it if necessary to avoid partial 
flap loss $[2-4,7,8]$.

The technique was indicated in patients with small or moderate volume breasts with or without ptosis. This included patients with moderate lateral breast skin and glandular defects where there was not enough breast tissue to perform the reconstruction by local glandular flaps or reduction mammaplasty [4]. We demonstrated the possibility of increasing options to perform this technique for the patients who want to correct soft tissue bulging of lateral thoracic area with breast reconstruction. Because of disproportionate excess skin of lateral chest wall, obese patients who underwent radical mastectomy, usually seek further aesthetic body contouring surgery to improve their body image. It is reliable method to construct an autogenous tissue reconstruction and shaping of lateral breast with reducing lateral chest wall excess.

The lateral thoracodorsal flap combined with an implant is a useful for breast reconstruction and satisfactory results can be obtained if a thorough preoperative plan is set up and implemented in appropriate patient such as this case. A surgeon has the possibility to achieve a good cosmetic and functional outcome with a low scar morbidity and few early complications.

\section{REFERENCES}

1. Holmström H, Lossing C. The lateral thoracodorsal flap in breast reconstruction. Plast Reconstr Surg 1986;77:933-43.

2. Woerdeman LA, van Schijndel AW, Hage JJ, et al. Verifying surgical results and risk factors of the lateral thoracodorsal flap. Plast Reconstr Surg 2004;113:196-203; discussion 204-5.

3. Yang JD, Ryu DW, Lee JW, et al. Usefulness of a lateral thoracodorsal flap after breast conserving surgery in laterally located breast cancer. Arch Plast Surg 2013;40:367-73.

4. Munhoz AM, Montag E, Arruda EG, et al. The role of the lateral thoracodorsal fasciocutaneous flap in immediate conservative breast surgery reconstruction. Plast Reconstr Surg 2006;117:1699-710.

5. Clough KB, Kroll SS, Audretsch W. An approach to the repair of partial mastectomy defects. Plast Reconstr Surg 1999;104:409-20.

6. Kroll SS, Singletary SE. Repair of partial mastectomy defects. Clin Plast Surg 1998;25:303-10.

7. Blomqvist L, Malm M. Clinical experience with the lateral thoracodorsal flap in breast reconstruction. Ann Plast Surg 1999;43:7-13.

8. Blomqvist L, Malm M, Holmström H, et al. The lateral thoracodorsal flap in breast reconstruction: a comparison between two plastic surgical centres. Scand J Plast Reconstr Surg Hand Surg 2000;34:327-30.

9. Hage JJ, Woerdeman LA. The design of the lateral thoracodorsal flap. Ann Plast Surg 2008;61:302-5. 